\title{
Impact of COVID-19 pandemic and lockdown on retinal detachments
}

\author{
Alicia Poyser $\mathbb{D}^{1} \cdot$ Sundeep S. Deol ${ }^{1} \cdot$ Lina Osman $^{1} \cdot$ Tharsica Sivagnanasithiyar $^{1} \cdot$ Helen J. Kuht ${ }^{2} \cdot$ Roslyn Manrique $^{1}$. \\ Linda O. Okafor ${ }^{1} \cdot$ David Sharpe $^{3} \cdot$ Vijay Savant $^{1} \cdot$ Usman Sarodia $^{1} \cdot$ Nagini Sarvananthan $^{1} \cdot$ Ray Chaudhuri $^{1}{ }^{1}$. \\ Somnath Banerjee ${ }^{1} \cdot$ Joyce Burns $^{1} \cdot$ Mervyn G. Thomas ${ }^{1,2}$
}

Received: 3 July 2020 / Revised: 7 August 2020 / Accepted: 10 August 2020 / Published online: 18 August 2020

(C) The Royal College of Ophthalmologists 2020

\section{To the Editor:}

We read with interest, the article 'The impact of COVID policies on acute ophthalmology services-experiences from Moorfields Eye Hospital NHS Foundation Trust' [1]. The paper emphasises disparity between attendance to A\&E prior-to and during the COVID-19 outbreak. Interestingly, the authors observe a reduction in the number of patients presenting with retinal detachments (RD) during COVID19 lockdown period. However, further analysis on status of the macula (on versus off), number of cases of posterior vitreous detachments (PVD) and retinal tears have not been described in the report.

At the University Hospitals Leicester, we compared presentations of vitreoretinal pathologies to our emergency eye department (EED) during the COVID-19 pandemic against an equivalent period in 2019. Data of EED attendance from electronic patient records were extracted over a 31-day period during: (study period 1 (SP1)) COVID-19 pandemic lockdown in UK (24th March 2020-23rd April 2020) and (study period 2 (SP2)) the equivalent 2019 period (24th March 2019-23rd April 2019). This included a total of 853 patients (403 females, 449 males; mean age $\pm \mathrm{SD}=$ $48.5 \pm 20.0$ years) from SP1, and 1818 patients' (969 females, 849 males; $49.0 \pm 22.1$ years) from SP2.

A 53\% reduction in EED attendance was noted between SP1 and SP2. We observed a $60 \%$ reduction in the number of retinal tears presenting to EED during SP1 $(n=6)$ compared to SP2 $(n=15)$. Similarly, we had a $64.9 \%$ reduction in PVD presenting between SP1 $(n=46)$ and SP2 $(n=131)$. We observed a $65.7 \%$ reduction in number of $\mathrm{RD}$ between SP1 and SP2. There was a significantly $\left(\mathrm{X}^{2}=\right.$ $6.66, p=0.0099)$ higher proportion of macula-off $\mathrm{RD}$ (84.6\% of all RD) during SP1 when compared to SP2 (42.9\%) (Fig. 1).

Our work supports the authors' initial observations and expands on this by showing higher proportion of maculaoff RD and lower number of retinal tears during SP1 suggestive of delayed presentation in these cases. This raises concerns about increased morbidity from ocular pathology unrelated directly to COVID19. We anticipate additional pressures on EED and vitreoretinal services due to complications and associated morbidity from delayed presentations.
Mervyn G. Thomas

mt350@le.ac.uk

1 Department of Ophthalmology, University Hospitals of Leicester, Leicester Royal Infirmary, Leicester LE1 5WW, UK

2 The University of Leicester Ulverscroft Eye Unit, Department of Neuroscience, Psychology and Behaviour, University of Leicester, RKCSB, PO Box 65, Leicester LE2 7LX, UK

3 IT services, University Hospitals of Leicester, Leicester Royal Infirmary, Leicester LE1 5WW, UK 


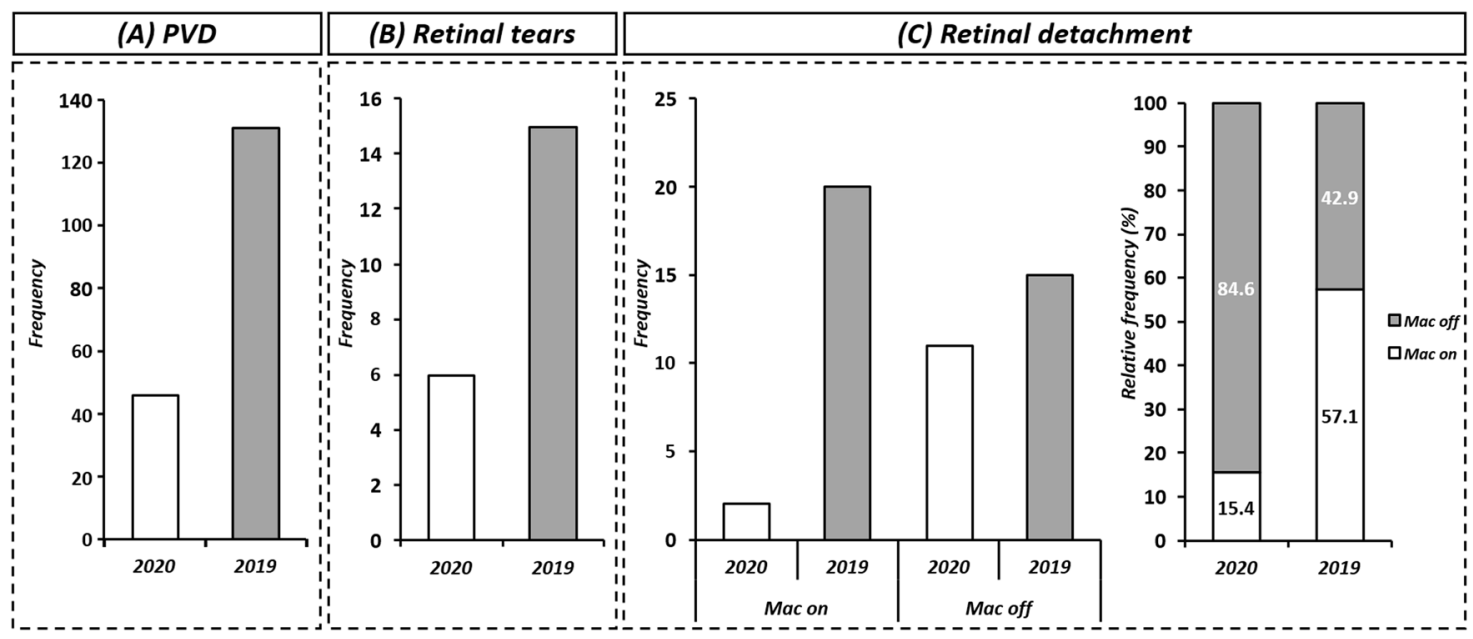

Fig. 1 Vitreoretinal pathologies presenting to the eye emergency department. Reduced number of cases were seen for posterior vitreous detachments (PVD) (a) retinal tears (b) and retinal detachment (c)

Funding HJK is supported by Medical Research Council (MRC), London, UK (grant number: MC_PC_17171). MGT is supported by National Institute of Health Research (NIHR) (CL-2017-11-003) and the Ulverscroft Foundation.

\section{Compliance with ethical standards}

Conflict of interest The authors declare that they have no conflict of interest. during COVID19 related lockdown in the UK compared against an equivalent period in 2019. The proportion of macula off retinal detachments was significantly higher during lockdown (c).

Publisher's note Springer Nature remains neutral with regard to jurisdictional claims in published maps and institutional affiliations.

\section{Reference}

1. Wickham L, Hay G, Hamilton R, Wooding J, Tossounis H, da Cruz L, et al. The impact of COVID policies on acute ophthalmology services - experiences from Moorfields Eye Hospital NHS Foundation Trust. Eye. 2020;34:1189-92. https://doi.org/10.1038/s41433-020-0957-2. 